\title{
The evoked cardiac response under processing load
}

\author{
ROBERT J. BARRY \\ University of New South Wales, Kensington, Australia
}

\begin{abstract}
Previous work has suggested that HR deceleration, commonly obtained in OR studies, re flected a preliminary process of stimulus registration rather than the OR itself. Research by the Laceys indicated that such a process was associated with a primary bradycardia, but they described this only over the first two poststimulus beats and not in an OR context. A preliminary study from this laboratory began the exploration of connections between these two phenomena by establishing their coexistence in an OR experiment. The present study aimed to examine the relationship between such HR deceleration and primary bradycardia in a complex cardiac response reflecting both stimulus and situational factors. The results suggest that the initial response of cardiac deceleration in the OR context may be nothing more than a primary bradycardia reflecting stimulus registration.
\end{abstract}

The phasic cardiac response evoked by a simple stimulus is a complex, and potentially multiphasic, response reflecting both stimulus parameters and situational factors. Phasic autonomic responses and their relation to stimulus parameters have received most attention in the context of Sokolov's distinction between orienting and defensive responses (ORs and DRs, respectively), although little reference was made to cardiac components in the classical literature. On the other hand, our notions of situational determinants in cardiac activity derive from J. I. Lacey's (1967) stimulus intake and rejection hypothesis: intake is accompanied by cardiac deactivation, rejection by cardiac activation. Graham and Clifton (1966) attempted to integrate these theories by identifying heart rate (HR) deceleration with the OR, and HR acceleration with the DR. Such an integration may be questioned on at least two grounds.

Velden and Schumacher (1979) have concentrated upon one of these: that Graham and Clifton's OR/DR distinction was empirically based on indifferent stimuli and emphasized the objective nature of the stimulus, while Lacey's concept of "situation" included the subject's intended response as well as the objective aspects of the stimulus. After carrying out a number of studies, Velden and Schumacher concluded that HR decelerations occurred when ORs might be expected, but that this response was "relatively weak ... [and may be] obscured by situational requirements that have an opposite effect on cardiac activity"' (p. 215). They contrasted this response with

My thanks are extended to Felicia Mitchell for assistance with data collection and analysis. Address reprint requests to Robert J. Barry, School of Education, University of New South Wales, Kensington, N.S.W. 2033, Australia. anticipatory decelerations that fitted "more easily into the theoretical frame of Lacey's approach" (p. 215) and emphasized the usefulness of the intentional aspect of Lacy's theory.

Numerous studies of information processing requirements have also supported this intentional aspect and its outcomes in the cardiac response. A recent review by Bohlin and Kjellberg (1979), although concentrating upon two-stimulus paradigms, covers much relevant material. In such a paradigm (as in a warned reaction-time task), it is common for the first member of the stimulus pair to elicit HR deceleration (D1) followed by acceleration (A) and a second deceleration (D2) before the second stimulus occurs. Bohlin and Kjellberg examined a number of hypotheses regarding the nature of these components in the cardiac response. They concluded that D1 could be considered as an OR to the first stimulus, whereas D2 might best be thought of as reflecting an expectancy process. While rejecting an OR/DR explanation for the A component, the authors reached no firm conclusions regarding alternative hypotheses. One hypothesis not ruled out is that $A$ reflects informationprocessing requirements (Coles \& Duncan-Johnson, 1975; Walter \& Porges, 1976). Such a view, derived from the Lacey position mentioned above, emphasizes again the different effects of the stimulus and situational factors. Bohlin and Kjellberg confirmed this in their conclusion that "the data ... are conclusive in definitely showing that the components reflect at least partly independent processes" (p. 193). Thus, the integration proposed by Graham and Clifton (1966) seems to have failed: we can discriminate components of complex evoked cardiac responses that appear in OR/DR situations from those that appear when stimulus intake/rejection (in Lacey's sense) is expected. 
Another ground for questioning Graham and Clifton's thesis is that a number of studies (e.g., Barry, 1977a, 1977b; Hulstijn, 1978; van Olst, Heemstra, \& ten Kortenaar, 1979) have indicated that the evoked cardiac response of HR deceleration produced by simple stimulation fails to habituate with stimulus repetition. Such a failure contradicts the view of HR deceleration as an OR indicator, since a defining characteristic of the OR, and necessarily of its indices, is habituation over trials. It is interesting to note that, in the studies by Velden and Schumacher discussed above, the cardiac response of $H R$ deceleration elicited by the tone alone (and identified by them as an OR) did not habituate over trials. Graham (1979) has attempted to integrate such responses into her theory by labeling nonhabituating cardiac decelerations as "subcortical ORs." Unfortunately, there is no independent criterion for such a distinction, and it would appear to be more parsimonious to accept that cardiac decelerations are not adequate indices of the $O R$.

Barry $(1981,1982$, in press) has presented a theory of preliminary processes in OR elicitation that accommodates a nonhabituating cardiac response. This theory was developed in response to the observed fractionation of phasic responses in the OR context, which had been an embarrassment to Sokolov's unitary OR concept. In the theory, a number of attributes of the stimulus are processed separately, and the outcome of these preliminary processes determines OR magnitude. The initial process is stimulus registration, in which the stimulus occurrence is registered as a precursor of the processing of stimulus magnitude and novelty: these last two processes parallel those of dual-process theory (Groves \& Thompson, 1970). Stimulus registration in the OR context of innocuous stimuli is hypothesized to be reflected in a HR deceleration that is independent of stimulus magnitude or novelty. Lacey and Lacey $(1977,1978,1980)$ have recently carried out extensive work on HR deceleration related to stimulus rather than situational characteristics which appears to be relevant in the present context. Brief stimuli produce an increase in pulse duration in the cardiac cycle in which stimulus onset occurs, and this effect is dependent upon the cycle time of stimulation. The earlier that stimulation occurs in a cycle, the greater is the slowing of that cycle; later stimulation produces proportionally less slowing of that cycle, but a greater slowing of the following cycle. Parallels with similar effects found in animal studies led the Laceys to consider this as a vagally mediated primary (or reflex) bradycardia, which they described as "an index of an early process of stimulus-registration by the nervous system" (1980, p. 22). Clearly, this primary bradycardia should be compatible with the stimulus registration process in preliminary process theory. Barry
(1983) looked for primary bradycardia in protocols from an earlier OR study, and found evidence that the two phenomena coexisted and were similarly independent of stimulus parameters, leading to the hypothesis that these two phenomena are aspects of the same process.

Barry (1983) noted that the Laceys had used stimuli of much shorter duration than was common in OR work $-100 \mathrm{msec}$ compared with the $2 \mathrm{sec}$ usually employed in our laboratory. Since temporal summation had been evidenced in animal studies of primary bradycardia, Barry (1983) viewed the HR response in the OR context as a cumulative primary bradycardia. However, the Laceys (Note 1) have pointed out that the temporal summation found in animal studies with punctate stimuli cannot be generalized to the continuous stimuli used in our OR work. This requires that the cardiac response in the OR context, which at least begins as a primary bradycardia, is either nothing more than primary bardycardia or involves some other (and as yet unknown) process. In this regard, it is unfortunate that the Laceys have not reported data beyond the cardiac cycle following stimulus onset.

This study was designed to examine the hypothesis that the cardiac response reflecting stimulus registration in the OR context is solely a primary bradycardia. It was noted above that in an informationprocessing context, Velden and Schumacher (1979) had characterized such a response as "relatively weak" and possibly "obscured by situational requirements." In fact, such influences should be additive and, in principle, separable. Accordingly, the present study imposed situational requirements that were aimed at producing a two-component cardiac response for analysis. In Bohlin and Kjellberg's (1979) systematization, a single stimulus was expected to elicit D1 (reflecting stimulus registration) preceding, and partly obscured by, A (reflecting processing requirements).

\section{METHOD}

\section{Subjects}

Twenty university students, aged between 20 and 25 years, volunteered to participate in the study as one means of fulfilling a course requirement in Educational Psychology at the University of New South Wales. There were 8 males and 12 females.

\section{Apparatus}

Stimuli consisted of $2400-\mathrm{Hz}$ tones in random combinations of either 33 or $50 \mathrm{~dB}$ re $20 \mu \mathrm{N} / \mathrm{m}^{2}$, with $.1-, 1.1-$, or $2.1-\mathrm{sec}$ duration, presented via circumaural headphones. Interstimulus interval varied randomly among 9,11 , and $13 \mathrm{sec}$. Stimuli were presented in four blocks, separated by 2 -min rest periods. The number of stimuli in each block varied randomly from 48 to 52 . The first 192 stimuli were used in the analyses. Stimulus presentation was controlled by an EXIDY microcomputer.

The EKG was recorded from standard lead 1 on a Stoelting Multiscribe. The amplified signal was used to trigger an R-wave
} 
peak detector (following Shimizu, 1978), the output of which was led to a second EXIDY microcomputer, which timed R-R intervals. The first microcomputer communicated information regarding stimulus presentations to the second. For each stimulus, the period of one prestimulus and five poststimulus beats (the first of which contained the stimulus onset) and the time from the last prestimulus $\mathbf{R}$-wave to stimulus onset were recorded on floppy disk for off-line analysis.

\section{Procedure}

Upon arrival at the laboratory, each subject was seated in a comfortable armchair and fitted with the EKG electrodes while the procedure was explained. The subject was told that a large number of low-level tones would be presented over the headphones, and that these would occur in four blocks, with the number in each block randomly varied by the computer, which controlled stimulus presentation. The stated task was to count the number of sounds heard over the headphones, irrespective of their loudness or duration. The instructions indicated that the experimenter would return after each block to quiz the subject on the number of tones counted, and promised 50 cents for each block correctly counted. The subject cubicle was then darkened and recording commenced shortly after. At the end of each block, the experimenter returned to the subject cubicle and paid a 50 cent coin if the count was correct. After a brief interval, the experiment continued in a similar fashion until four blocks were completed. Subjects were included in the study if they obtained at least three correct block totals.

\section{RESULTS}

\section{Preliminary Data Reduction}

For each stimulus presentation, the interval from the last prestimulus $R$-wave to stimulus onset was used to determine in which quartile of the cardiac cycle stimulation commenced. This quartile value, together with the stimulus parameters of intensity (33 or $50 \mathrm{~dB})$ and duration $(.1,1.1$, or $2.1 \mathrm{sec})$, was used to collapse the data within subjects to form 24 mean beat-by-beat response profiles of $R-R$ periods over six beats from prestimulus beat 1 to poststimulus beat 5 . The means of these values, expressed as deviations from the prestimulus period, are shown in Figure 1. Inspection of this figure suggests that the cardiac response in this study consisted of a small deceleratory HR response (D1), partly obscured by a larger acceleratory HR response (A), as expected from previous work. Figure 1 also includes a hypothetical model of each of these components. These add to form the observed response.

\section{Evidence for Two Components}

The model responses shown in Figure 1 suggest that D1 might be well described by quadratic and cubic trends over the first five poststimulus beats. This is generally compatible with the deceleratory HR response reported by Barry (1983), which appeared as a significant quadratic trend over a similar interval. However, a quadratic trend alone would not allow the manifestation of any durational effects in D1, and the cubic trend was included for this reason. In contrast, $\mathbf{A}$ appears to consist largely of a linear

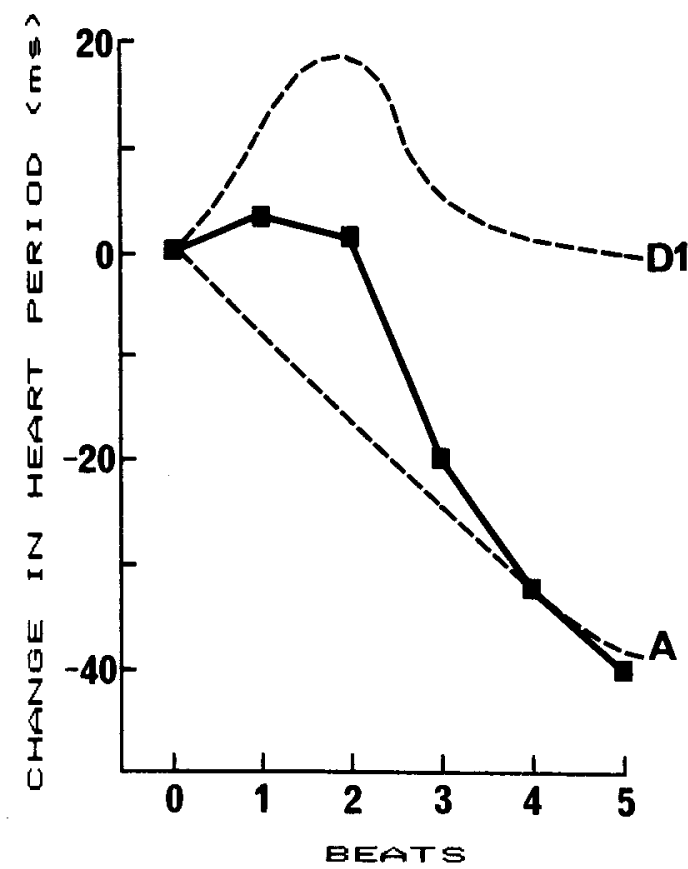

Figure 1. The points connected by heavy lines indicate mean changes in heart period from the prestimulus beat (labeled 0 ) for each of the five poststimulus beats. The broken lines represent models of D1 and A, which add to produce the observed response.

decrease in period over this interval, that is, a linear trend over beats.

The 144 data points for each subject were submitted to a four-way repeated-measures ANOVA over stimulus intensity and duration, cardiac phase at a stimulus onset, and beats. Within beats, linear, quadratic, and cubic trends were examined as models of A (linear trend) and D1 (quadratic and cubic trends). The linear trend was significant ${ }^{1}[F(1,19)=42.82$, MSe $=17,821.9521$, as were the quadratic and cubic trends $[\mathrm{F}(1,19)=39.04$, $\mathrm{MSe}=1,367.760$, and $\mathrm{F}(1,19)=$ 27.44, $\mathrm{MSe}=1,739.083$, respectively]. Together, these three trends accounted for $98.7 \%$ of the systematic variance within beats.

\section{Effects of Variables on Components}

In the four-way ANOVA, the effects of the variables of stimulus duration and intensity, and of cardiac phase at stimulus onset, upon the two components, D1 and A, may be found in the interactions between these variables and the three trends over beats. For D1, interactions with the quadratic and cubic trends were examined; for $\mathbf{A}$, interactions with linear trend were examined.

D1. Some effect of the quartile of stimulus occurrence was apparent, in that the interaction of quadratic trend with quartile approached significance $[F 3,57)=2.73, \mathrm{MSe}=915.741$; cf. critical $F=2.76]$. Figure 2 shows response curves of raw period changes over beats for each cardiac quartile at which stimulus onset occurred. Figure 3 shows derived models of D1 


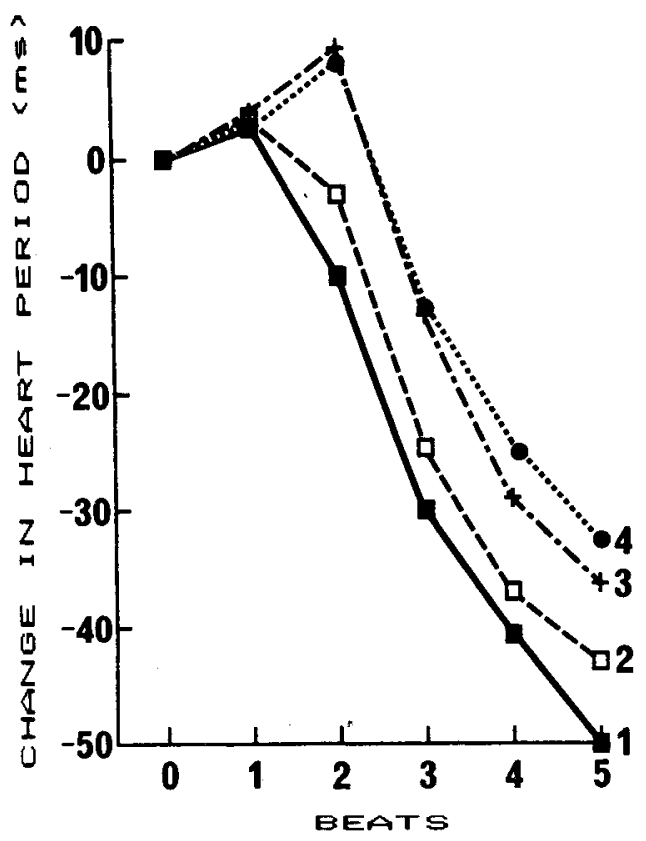

Figure 2. Mean changes from prestimulus heart perlod as a function of beats for stimull presented at each quartile of the cardiac cycle. The quartile is Indicated by the numbers 1 to 4 at the right of the figure.

and $A$ for each cardiac phase. The models of D1 were each derived from the raw curves of Figure 2 as the weighted sum of the quadratic and cubic regression lines over the corresponding means, adjusted to pass through appropriate zero points. The zero point was set at the prestimulus point for stimuli presented in the first quartile, and one quarter of a cycle later for each succeeding quartile curve, since no response could commence prior to stimulus onset. These curves suggest that the peak of D1 tends to appear later as stimulus onset is delayed, and that D1 may be followed by another deceleratory HR component after its completion. No other effects or interactions with the quadratic or cubic trends approached significance. Of particular importance in these negative results is the absence of an effect of stimulus duration. Figure 4 shows derived models of D1 for each value of stimulus duration. Although there is a suggestion of some effect in Figure 4, in that longer stimuli appear to produce more HR deceleration later in the response, this effect failed to reach significance $[\mathrm{F}(2,38)=0.27, \mathrm{MSe}=575.939$, for quadratic trend, and $\mathrm{F}(2,38)=1.65, \mathrm{MSe}=502.194$, for cubic trend].

A. The interaction of linear beats with cardiac phase was significant $[\mathrm{F}(3,57)=4.67, \mathrm{MSe}=1,296.279]$. This is shown in the bottom part of Figure 3 by the linear regression lines over means, adjusted to pass through the appropriate prestimulus points as defined for D1 above. It would appear that, in addition to a simple delay effect (also apparent in the raw curves in Figure 2), later stimulus onset may produce less $\mathrm{HR}$ acceleration than stimuli occurring early in the cardiac cycle. No other effects or interactions with A were obtained.

\section{Primary Bradycardia}

Evidence for primary bradycardia with the characteristics established by the Laceys was sought in the derived curves for D1, as a function of cardiac phase at stimulus onset, shown in Figure 3. The slowing of the cardiac cycle containing stimulus onset, and the following cycle, are shown as functions of the quartile of stimulus onset in Figure 5. This appears to indicate that the earlier the stimulation commences in the cycle, the greater is the slowing of that cycle (beat 1), whereas later stimulation has its greatest effect in slowing the following cycle (beat 2). This is the defining characteristic of the Laceys' primary bradycardia. The significance of this interaction between the quartile effect and beat was examined in the data underlying the mean curve shown in Figure 1, which was based on deviations from the prestimulus period. An ANOVA similar to that reported above, but including only the first two poststimulus beats, indicated that the interaction between beat and quar-

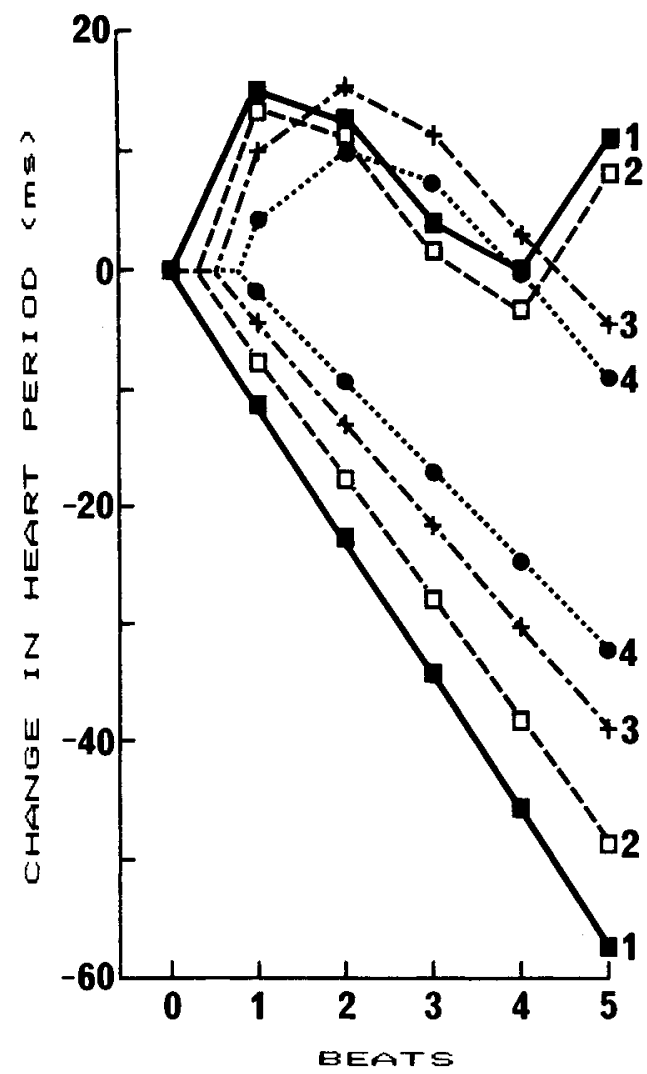

Figure 3. Models of D1 (top four curves) and A (bottom four curves), derived from the changes in period over beats, as a function of the quartile of the cardiac cycle which contained the stimulus onset (shown on the right of each curve). D1 is the weighted sum of quadratic and cubic trends over beats, while $A$ is the linear trend component. 


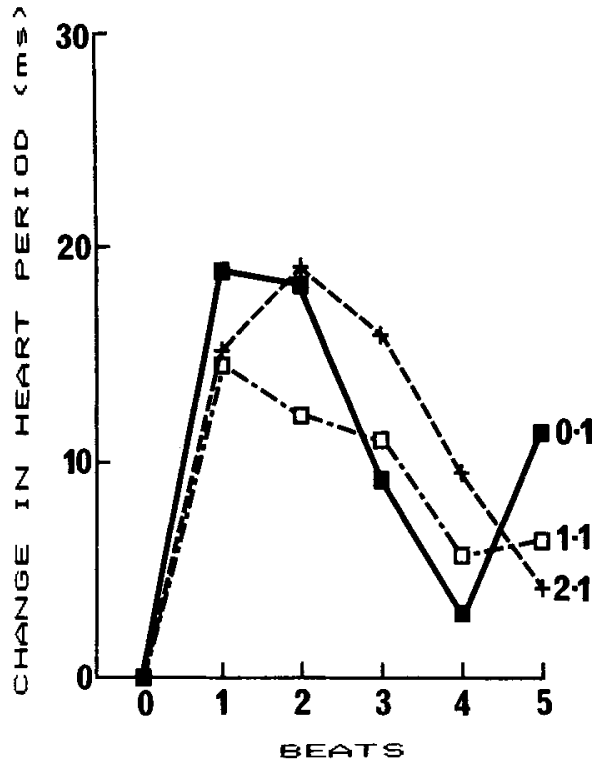

Figure 4. Models of D1 over beats for the three stimulus durations (shown on the right of each curve).

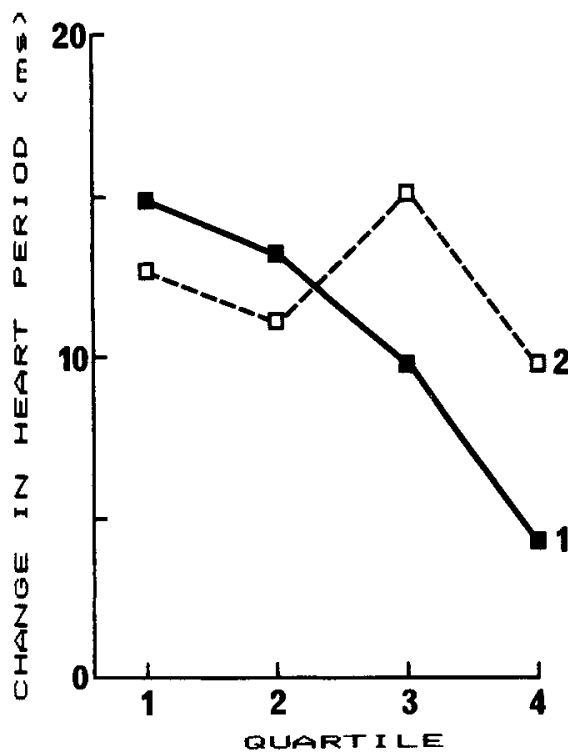

Figure 5. Increases in heart period from the prestimulus value for the cycle containing the stimulus onset (labeled 1 in the figure) and for the subsequent cycle (labeled 2), as a function of the cardiac phase at stimulus onset.

tile was significant $[\mathrm{F}(3,57)=7.55, \mathrm{MSe}=856.477]$. This confirms the existence of primary bradycardia in D1 in this study. No other effects or interactions approached significance.

\section{DISCUSSION}

As expected from previous work, the cardiac response obtained in this study consisted of a complex biphasic response of HR deceleration (D1) followed by acceleration (A). The first of these components is identifiable as the response commonly obtained in the OR context and considered by preliminary process theory to reflect stimulus registration. The second component may be taken to reflect informationprocessing load, due here to the necessity of identifying relatively quiet tones of differing durations as countable stimuli and of accumulating that count. In analyzing these components, D1 was modeled as a combination of quadratic and cubic trends over the first five poststimulus beats, and $\mathbf{A}$ was considered as a linear trend over these beats. These three trends, and hence the two independent components modeled by them, were statistically significant.

Cardiac activation, the A component, was found to be significantly affected by the cardiac phase at stimulus onset. Over the five poststimulus beats, delay in stimulus presentation appears to result in a slowing of the rate of HR increase (see Figure 3). Although this may be due in part to a simple delay in the onset of the underlying process, time-locked to the delay in stimulus onset, it may reflect also an interference between that process and the cardiac event itself. However, such an effect would be contrary to previous findings that cardiac phase at stimulus onset failed to affect performance in either simple reaction time (RT) (Thompson \& Botwinick, 1970) or choice RT tasks (Jennings \& Wood, 1977), or auditory sensitivity and response bias in a signaldetection paradigm (Delfini \& Campos, 1972). No other effects were found in A, a result that was not unexpected from the experimental manipulations employed. Since subjects were included in the study only if they obtained at least three correct block totals, it is reasonable to assert that all stimuli were readily discriminable. Thus, identification and counting, considered as processing load, would not be expected to vary systematically over the stimulus parameters examined here.

Some effect of the quartile of stimulus onset was also apparent in D1. It is suggested that the peak of HR deceleration may occur later as the cardiac phase of stimulation is delayed, a simple effect reflecting time-locking of the response with stimulus onset. There is also some suggestion that completion of D1 may be followed by another deceleratory HR response, and this possibility is currently under investigation in my laboratory. Of the more immediate relevance is the finding that stimulus durations ranging from 0.1 to $2.1 \mathrm{sec}$ produced essentially identical versions of D1. There is some suggestion that longer stimuli produced more HR deceleration later in the response, but this failed to reach significance. Figure 4 indicates that this effect is relatively small, so that even if we were to accept its existence, its contribution to the response form could not be considered major.

The models of D1 for stimuli commencing at different phases of the cardiac cycle (Figure 3) provided evidence for a primary bradycardia consistent with 
the properties cited by the Laceys. Figure 5 indicates the significant interaction between the quartile effect and beat that was derived from these models. There was no difference in this interaction for stimuli of different durations, indicating that the primary bradycardia, at least over the first two poststimulus beats, was independent of stimulus duration.

These results suggest that D1 is little more than primary bradycardia. First, the cardiac slowing over the first two beats, for all durations, is identifiable as the Laceys' primary bradycardia. Second, beyond these first two beats, there is a return towards baseline, suggesting recovery as the only major influence on HR deceleration after the primary bradycardia of the first two poststimulus beats. Third, there is no appreciable difference between the HR deceleration elicited by the $0.1-\mathrm{sec}$ stimuli, which the Laceys discussed only in terms of primary bradycardia, and that elicited by the stimuli of greater duration. For these reasons, it appears that D1, the deceleratory HR response in the OR context, may be considered as a primary bradycardia.

The integration achieved by such an identification is of some importance, particularly to the development of preliminary process theory. The concept of the evoked cardiac response functioning as an indicator of the preliminary process of stimulus registration was derived from observations that the response appeared to be relatively independent of stimulus parameters within the OR context. The Laceys reached the same conclusion, regarding the role of primary bradycardia, from a different perspective, emphasizing its physiological basis and rapid onset. The present results, and the consequent integration of these two areas, provide support for the concept of stimulus registration as a separable stage in elicitation of the OR, and for the use of cardiac deceleration as its index. Such a conclusion refers only to the initial HR deceleration following stimulus onset, since later decelerations may reflect other aspects of processing demands sensitive to situational manipulations.

\section{REFERENCE NOTE}

1. Lacey, B. C., \& Lacey, J. I. Personal communication, 1982.

\section{REFERENCES}

BARRY, R. J. The effect of "significance" upon indices of Sokolov's orienting response: A new conceptualization to replace the OR. Physiological Psychology, 1977, 5, 209-214. (a)

BARRY, R. J. Failure to find evidence of the unitary OR concept with indifferent low-intensity stimuli. Physiological Psychology, 1977, 5, 89-96. (b)

BARry, R. J. Signal value and preliminary processes in OR elicitation. Pavlovian Journal of Biological Science, 1981, 16, 144150.

BARRY, R. J. Novelty and significance effects in the fractionation of phasic OR measures: A synthesis with traditional OR theory. Psychophysiology, 1982, 19, 28-35.
Barry, R. J. Primary bradycardia and the evoked cardiac response in the OR context. Physiological Psychology, 1983, 11, 135-140.

BARRy, R. J. Preliminary processes in OR elicitation. Acta Psychologica, in press.

Bohlin, G., \& KJELlberg, A. Orienting activity in two-stimulus paradigms as reflected in heart rate. In H. D. Kimmel, E. H. van Olst, \& J. F. Orlebeke (Eds.), The orienting reflex in humans. Hillsdale, N.J: Erlbaum, 1979.

Coles, M. G. H., \& Duncan-Johnson, C. C. Cardiac activity and information processing: The effects of stimulus significance and detection and response requirements. Journal of Experimental Psychology: Human Perception and Performance, 1975, 1, 418-428.

Delfini, L. F., \& Campos, J. J. Signal detection and the "cardiac arousal cycle." Psychophysiology, 1972, 9, 484-491.

Graham, F. K. Distinguishing among orienting, defense, and startle reflexes. In H. D. Kimmel, E. H. van Olst, \& J. F. Orlebeke (Eds.), The orienting reflex in humans. Hillsdale, N.J: Erlbaum, 1979.

Graham, F. K., \& Clifton, R. K. Heart-rate change as a component of the orienting response. Psychological Bulletin, 1966, 65, 305-320.

Groves, P. M., \& Thompson, R. F. Habituation: A dual-process theory. Psychological Review, 1970, 77, 419-450.

HulstiJn, W. Habituation of the orienting response as a function of arousal induced by three different tasks. Biological Psychology, 1978, 7, 109-124.

Jennings, J. R., \& Wood, C. C. Cardiac cycle time effects on performance, phasic cardiac responses, and their intercorrelation in choice reaction time. Psychophysiology, 1977, 14, 297-307.

LACEY, B. C., \& LACEY, J. I. Change in heart period: A function of sensorimotor event timing within the cardiac cycle. Physiological Psychology, 1977, 5, 383-393.

LACEY, B. C., \& LACEY, J. I. Two-way communication between the heart and the brain: Significance of time within the cardiac cycle. American Psychologist, 1978, 33, 99-113.

Lacey, B. C., \& LaceY, J. I. Cognitive modulation of timedependent primary bradycardia. Psychophysiology, 1980, 17, 209-221.

LACEY, J. I. Somatic response patterning and stress: Some revisions of activation theory. In M. H. Appley \& R. Trumbull (Eds.), Psychological stress: Issues in research. New York: Appleton-Century-Crofts, 1967.

Shimizu, H. Reliable and precise identification of $\mathbf{R}$-waves in the EKG with a simple peak detector. Psychophysiology, 1978, 15, 499-501.

Thompson, L. W., \& Botwinick, J. Stimulation in different phases of the cardiac cycle and reaction time. Psychophysiology, $1970,7,57-65$.

van Olst, E. H., Heemstra, M. L., \& ten Kortenaar, T. Stimulus significance and the orienting reaction. In $H$. D. Kimmel, E. H. van Olst, \& J. F. Orlebeke (Eds.), The orienting reflex in humans. Hillsdale, N.J: Erlbaum, 1979.

Velden, M., \& Schumacher, R. Orienting and defensive cardiac responses. In H. D. Kimmel, E. H. van Olst, \& J. F. Orlebeke (Eds.), The orienting reflex in humans. Hillsdale, N.J: Erlbaum, 1979.

Walter, G. F., \& Ponges, S. W. Heart rate and respiratory responses as a function of task difficulty: The use of discriminant analysis in the selection of psychologically sensitive physiological responses. Psychophysiology, 1976, 13, 563-571.

\section{NOTE}

1. Significance level used is $p<.05$.

(Manuscript received September 6, 1983; accepted for publication December 1,1983 .) 